\title{
Miniaturlautsprecher aus dem Drucker
}

\author{
In einem additiven Fertigungsverfahren lassen sich Miniaturlautsprecher als Teil von \\ piezoelektrischen, mikroelektromechanischen Systemen effizient und kostengünstig \\ herstellen. Dies beweisen Wissenschaftler im Projekt ,Generator'.
}

Piezo-MEMS, piezoelektrische, mikroelektromechanische Systeme, sind wahre technische Alleskönner, denn die hauchdünnen piezoelektrischen Schichten erfüllen wahlweise aktorische oder sensorische Funktionen: Entweder dehnen sie sich beim Anlegen eines elektrischen Feldes aus oder sie wandeln mechanische Bewegung in elektrische Spannung um. Entsprechend gefragt sind sie in der Kommunikations- oder Medizintechnik zum Beispiel als Sensor oder Aktor in Pumpen, Ventilen oder Lautsprechern - jeweils im Miniaturformat. Die Dünnschichten bestehen in der Regel aus Blei-ZirkonatTitanat (PZT), der derzeit leistungsfähigsten piezoelektrischen Funktionskeramik. Es kommen bevorzugt piezoelektrische, wenige $\mu \mathrm{m}$ dünne Schichten zum Einsatz, welche sich beispielsweise durch Ätzen oder direktes Drucken sehr genau strukturieren lassen.

\section{Laserunterstützte Druckverfahren}

Bislang wurden in der Produktion von Piezo-MEMS konventionelle vakuum- und maskenbasierte Herstellungsmethoden eingesetzt, die allerdings insbesondere bei der Produktion von Kleinserien sehr zeit- und kostenintensiv sind. Im Rahmen des vom Bundesministerium für Bildung und Forschung (BMBF) geförderten Projekts, Generator' entwickelte das Fraunhofer ILT gemeinsam mit dem Fraunhofer ISIT und dem IWE2 der RWTH Aachen daher als günstige Alternative eine Verfahrenskombination aus digitalem Tintenstrahldruck und Laserkristallisation: Nach dem Auftragen von PZT-Spezialtinte auf 8 “-SiliziumWafern folgt die Kristallisation mittels Laserstrahlung bei lokalen Temperaturen von über $700^{\circ} \mathrm{C}$. Für Qualität sorgt ein tem- peraturgeregelter Prozess, der die Temperaturschwankungen auf $\pm 5^{\circ} \mathrm{C}$ begrenzt.

\section{Trend zum Multi-Material- Stack}

Aus mehreren 20 bis $30 \mathrm{~nm}$ dünnen PZTSchichten wird ein mehrlagiger Aktuator mit einer Gesamtschichtdicke von 2 bis $3 \mu \mathrm{m}$ aufgebaut. „Anfangs brachten wir nur eine einfache Schicht auf, mittlerweile entsteht Schicht um Schicht ein MultiMaterial-Stack“, so ILT-Wissenschaftler Samuel Fink. Abwechselnd bauen sich übereinander insgesamt bis zu 30 Schichten aus Funktionskeramik- und Elektroden zu einem Mikrolautsprecher auf (Bild 1). Dank dieser Konstruktion soll der Aktuator eine bessere Performance und höhere Wiedergabequalität als übliche Aktuatoren aufweisen. Dabei greifen PZTSchichten und Elektroden-Schichten wie zwei sehr feine Kämme ineinander. Durch die schnelle Laserbearbeitung der Schichten sinkt die sonst minutenlange Bearbeitungszeit je Schicht auf wenige Sekunden. Als Elektrodenmaterial verwenden die Wissenschaftler statt des gängigen und sehr teuren Platins die elektrisch leitende Keramik Lanthan-Nickel-Oxid (LNO). Durch den Verzicht auf metallische Komponenten kann die Haltbarkeit dieser rein keramischen Multi-Material-Stacks deutlich gesteigert und die Materialkosten gleichzeitig gesenkt werden.

Legt man nun eine Wechselspannung an diesen Multi-Material-Stack an, verformen sich die PZT-Schichten in Bruchteilen von Sekunden und regen dadurch den ganzen Stack zur Schwingung an. Da das ganze System nur wenige $\mu \mathrm{m}$ dick ist und dadurch eine sehr geringe Masse hat, lassen sich so akustische Signale vor allem im Hochton-Bereich ausgezeichnet übertragen.

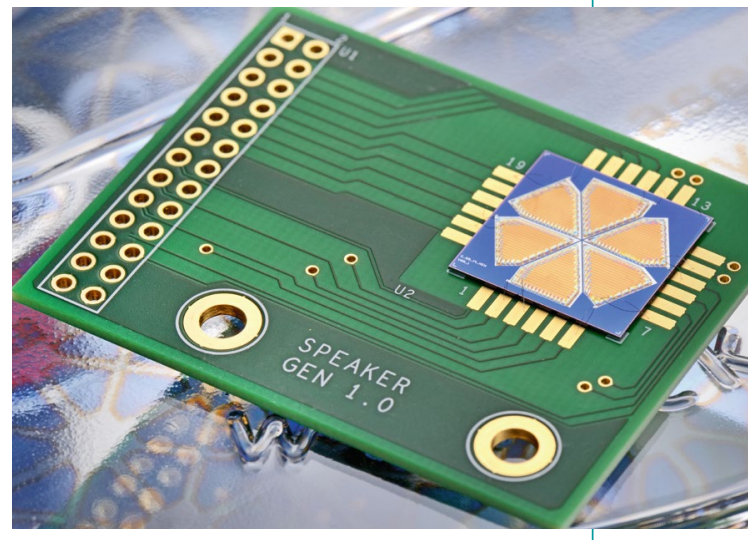

Bild 1 Sechseckig, winzig, günstig: Der MiniLautsprecher beweist, dass sich Mikroaktorik per Tintenstrahldrucker und Laser sekundenschnell und preiswert herstellen lässt. (๑ Fraunhofer ILT)

\section{Chance für kleine und} mittlere Unternehmen

Konventionelle Anlagen zum Herstellen von Dünnschicht-Elektronik kosten mehrere Millionen Euro und lohnen sich daher nur für die Großserienproduktion. Bei kleineren Losgrößen wird das additive Hybridverfahren interessant, vor allem wenn das Bauteil wie der Mikrolautsprecher aus mehreren Schichten besteht. Das Verfahren eignet sich daher besonders für kleine und mittlere Unternehmen, denn die Investition in die Anlagentechnik fällt im Vergleich zu konventioneller Technik deutlich günstiger aus. Fink: „Der Anwender benötigt dazu entsprechende Druck- und Lasersystemtechnik sowie speziell angepasste PZT- und LNOTinten. Sogar sehr kleine Job-Shops könnten sich daher in Zukunft eine Kleinserienproduktion für Mikroaktorik aufbauen."

Kontakt:

Fraunhofer-Institut für Lasertechnik ILT, 52074 Aachen, www.ilt.fraunhofer.de 